\title{
EQUILIBRIUM WITH FIXED BUDGETS AND SUPERLINEAR CONNECTIONS
}

\author{
A. M. RUBINOV ${ }^{1}$ and B. M. GLOVER ${ }^{1}$
}

(Received 17 May 1997; revised 5 April 1998)

\begin{abstract}
We study models of economic equilibrium with fixed budgets and assuming superlinear connections between consumption and production. Extremal problems and the existence of equilibria are discussed for such models along with some related differential properties. Examples to illustrate the broad nature of the model are discussed.
\end{abstract}

\section{Introduction}

The mathematical theory of economic equilibria forms an important part of mathematical economics. Fixed point theorems together with methods of convex analysis are used in the study of existence of equilibria in various economic situations (see, for example, $[1,2,6-8])$. There is a special class of models of economic equilibria whose analysis can be undertaken only by the application of simple convex optimization problems without fixed point theory (see, for example, [7]). This class is characterized by two assumptions: first, that the budgets of economic agents are fixed (that is, they do not depend on activity); and second, that the utility functions of agents are positively homogeneous.

Models of economic equilibrium with fixed budgets and positively homogeneous utility play an important role in the study of special models of economic dynamics (see [7]). One of the main problems in the theory of von Neumann-type models of economic dynamics is the construction of efficient trajectories. If a model describes the joint activity of various agents then the most natural mechanisms for designing efficient trajectories are equilibrium mechanisms. It has transpired that the usual Walras-type equilibrium models are not suitable for this purpose (see [7]). Construction of an efficient trajectory is possible only if the rates of growth of welfare are equal for all

'School of Information Technology and Mathematical Sciences, University of Ballarat, Australia.

(C) Australian Mathematical Society 2001, Serial-fee code 0334-2700/01 
agents so the agents have to subsequently redistribute their income. After redistribution each of the agents receives a new fund which can be considered as a fixed budget. Thus an equilibrium model with fixed budgets appears to be a natural tool for the construction of trajectories. Since von Neumann type-dynamics are described by positively homogeneous mappings it follows that the associated utility functions of these equilibrium models are also positively homogeneous.

Equilibrium mechanisms with fixed budgets used for the design of trajectories have been studied in detail in [7] only for the classical situation where it is assumed that there are no restrictions on free exchange. Equilibrium with restrictions on free exchange is studied in [5] and [7] in the framework of models with superlinear connections. The construction of efficient trajectories, by using equilibrium mechanisms with restrictions on free exchange, is much more complicated than without any restrictions. We have to investigate at least two problems before we can begin to study the action of equilibrium mechanisms. The first is to investigate the properties of equilibrium models with fixed budgets and positively homogeneous utilities under restrictions on free exchange and the second is to find mechanisms for redistribution of income between agents.

We shall study in detail the first problem in this paper. We suppose that restrictions on free exchange are expressed in the form of superlinear connections so we shall study models with fixed budgets and superlinear connections. We suppose also that an equilibrium for these models is defined in an abstract form by so-called matched four-tuples of vectors (see $[5,7])$. This abstract definition allows us to formulate meaningful definitions of equilibria for specific models. We verify this formulation for special models in this paper. In particular we give a definition of equilibrium for a model with public goods assuming that the superlinear connections are expressed through a matrix equality. (In fact matrix equalities are only used for the description of restriction in several interesting cases: exchange under fixed prices, direct distribution and mixed mechanisms (a mixture of free exchange and direct distribution)). Some results in this direction have been obtained in [11] but only for a model with fixed prices.

Without any restriction on free exchange, the main property of models with fixed budgets and positively homogeneous utilities is that (see $[7,9,12])$ it is possible to construct equilibria by solving a very simple convex extremal problem. We show that this assertion is also valid for models with some restriction on exchange. It is impossible to generalize the proofs given in $[7,9,12]$ for such models, so we develop a new approach to study this problem. This result allows us in particular to prove the existence of equilibria. We also study differential properties of equilibria and show that there is a system of $m$ first order differential equations with respect to $m$ variables such that the equilibrium utilities of the agents satisfy this system. It is very interesting that this system does not depend on the specific superlinear connection which is used 
in the definition of equilibria.

This paper has the following structure: Section 2 contains preliminary information on superlinear mappings. Section 3 is devoted to the definition of equilibria for a model with fixed budgets in the presence of restrictions on free exchange in the form of superlinear connections. Models with public goods are considered in Section 4. Extremal properties and the existence of equilibria are discussed in Section 5. Differential properties of equilibria are described in Section 6 and we conclude with a discussion of some open problems in the final section.

\section{Preliminaries on superlinear mappings}

In this section we provide some preliminary definitions and results on superlinear mappings which we need in subsequent sections.

Let $I$ be a finite set. We will use the following notation.

- $\mathbb{R}^{I}$ is the set of all functions defined on $I$ (that is, $I$ - vectors).

- $x^{i}$ is the $i$-th coordinate of a vector $x \in \mathbb{R}^{l}$.

- $x \geq y$ if $x^{i} \geq y^{i} \forall i \in I ; x>y$ if $x \geq y$ and $x \neq y ; x \gg y$ if $x^{i}>y^{i} \forall i \in I$.

- $[x, y]$ is the inner product of vectors $x$ and $y$. (We suppose that $\mathbb{R}^{I}$ is Euclidean space with the usual inner product.)

- $\mathbb{R}_{+}^{I}=\{x \in \mathbb{R}: x \geq 0\}$.

We shall use the following definitions (see [7] for a detailed discussion).

DEFINITION 2.1. A multivalued mapping $A: \mathbb{R}_{+}^{l} \rightarrow \mathbb{R}_{+}^{l}$ with $A(x) \neq \emptyset$ for all $x \in \mathbb{R}_{+}^{I}$ is called superlinear if $\operatorname{gr} A=\left\{(y, x) \in \mathbb{R}_{+}^{I} \times \mathbb{R}_{+}^{I}: x \in A(y)\right\}$ is a convex cone.

Using the terminology of convex analysis we can say that a superlinear mapping $A$ is a convex process such that $\operatorname{dom} A=\mathbb{R}_{+}^{I}$ and $\operatorname{Im} A \subseteq \mathbb{R}_{+}^{I}$. We will consider only closed superlinear mappings $A$ (that is, mappings where gr $A$ is a closed set) with the property $A(0)=\{0\}$.

DEFINITION 2.2. A superlinear mapping $A$ is called polyhedral if its graph is a polyhedral set.

In the following work we will use the superlinear polyhedral mapping $\mathscr{D}$ of free exchange which describes exchange between participants of an economic system provided that there are no restrictions on exchange. We assume that $m$ participants operate in the system and let $J=\{1, \ldots, m\}$. 
DEFINITION 2.3. The free exchange mapping $\mathscr{D}$ defined on the cone $\left(\mathbb{R}_{+}^{I}\right)^{J}=\mathbb{R}_{+}^{I^{m}}$ is given by the formula

$$
\mathscr{D}(y)=\left\{x=\left(x_{j}\right)_{j \in J} \in\left(\mathbb{R}_{+}^{\prime}\right)^{J}: \sum_{j \in J} x_{j}=\sum_{j \in J} y_{j}\right\} \quad \text { for } y=\left(y_{j}\right)_{j \in J} \in\left(\mathbb{R}_{+}^{\prime}\right)^{J}
$$

DEFINITION 2.4. Let $A: \mathbb{R}_{+}^{I} \rightarrow \mathbb{R}_{+}^{I}$ be a superlinear mapping. Then

(1) The mapping $A^{*}: \mathbb{R}_{+}^{I} \rightarrow \mathbb{R}_{+}^{I}$ is a conjugate with respect to $A$ if for all $f \in \mathbb{R}_{+}^{I}$ we have

$$
A^{*}(f)=\left\{g \in \mathbb{R}_{+}^{\prime}:\left(\forall y \in \mathbb{R}_{+}^{l}\right),(\forall x \in A(y))[f, x] \leq[g, y]\right\} ;
$$

(2) A four-tuple $(y, x, g, f)$ is called a matched four-tuple with respect to the mapping $A$ if

$$
x \in A(y), \quad g \in A^{*}(f), \quad[g, y]=[f, x] .
$$

We will require the following assertion (see [7]).

THEOREM 2.1. If $A: \mathbb{R}_{+}^{I} \rightarrow \mathbb{R}_{+}^{I}$ is a superlinear mapping then

$$
\max \{[f, x]: x \in A(y)\}=\inf \left\{[g, y]: g \in A^{*}(f)\right\} \text {. }
$$

If, in addition, $A$ is a polyhedral mapping then the infimum in this formula is attained.

\section{Equilibrium with fixed budgets and superlinear connections}

In this section we describe an equilibrium model with fixed budgets and superlinear connections. It is assumed that $m$ agents are active in the economy. We will denote the set $\{1, \ldots, m\}$ by $J$.

States of agents and states of the economy: Each agent $j \in J$ possesses a consumption set $X_{j}$ and a production set $Y_{j}$. It is assumed that $X_{j}=\mathbb{R}_{+}^{I}$ and $Y_{j} \subset \mathbb{R}_{+}^{I}$ is a compact convex set (here $I$ is the finite set of products). A state of the agent $j$ is represented by a vector $z_{j}=\left(x_{j}, y_{j}\right) \in \mathbb{R}_{+}^{I} \times Y_{j}$; a state of the system is a collection $\left(z_{j}\right)_{j \in J}$ where $z_{j}$ is a state of the agent $j$ for each $j \in J$.

Prices: Each agent is assumed to have their own prices, with purchase and sale prices not necessarily being equal. Let $p_{j}=\left(f_{j}, g_{j}\right)$ be prices used by the $j$-th agent, where $f_{j}$ are prices for purchased goods and $g_{j}$ are prices for sold goods. It is assumed that $f_{j}, g_{j} \in \mathbb{B}_{+}^{I}$. The vector $p=\left(p_{j}\right)_{j \in J}$ will be referred to as the price vector in the system. 
Preferences: The preferences of the $j$-th agent are described by a utility function $u_{j}$ defined on $\mathbb{R}_{+}^{I}$. We shall assume that $u_{j}$ is a nonnegative upper semicontinuous and superlinear (that is concave and positively homogeneous of degree one) function with the property that there is an interior point $x_{j}$ of the cone $\mathbb{R}_{+}^{\prime}$ such that $u_{j}\left(x_{j}\right)>0$. It follows immediately from superlinearity of $u_{j}$ that $u_{j}(x)>0$ on the interior of this cone.

Budgets: Each agent $j \in J$ has a certain budget $\lambda_{j}>0$ that can be spent in order to buy products.

The model $\mathscr{E}$ with fixed budgets is defined as

$$
\mathscr{E}=\left\{I, J,\left(Y_{j}\right)_{j \in J},\left(u_{j}\right)_{j \in J},\left(\lambda_{j}\right)_{j \in J}\right\} .
$$

In order to define an equilibrium for the model $\mathscr{E}$ we need a closed superlinear mapping $\mathscr{B}:\left(\mathbb{R}_{+}^{l}\right)^{J} \rightarrow\left(\mathbb{R}_{+}^{l}\right)^{j}$ with $\mathscr{B}(0)=\{0\}$. This mapping describes all possible exchanges among agents. More precisely if $y=\left(y_{j}\right)_{j \in J}$ with $y_{j} \in Y_{j}$ is a vector of manufactured products then a vector $x=\left(x_{j}\right)_{j \in J}$ is admissible for consumption if and only if $x \in \mathscr{B}(y)$.

Let $z=\left(\left(x_{j}, y_{j}\right)\right)_{j \in J}$ be a state of the model and $p=\left(\left(f_{j}, g_{j}\right)_{j \in J}\right)$ be a price vector. We shall use the notation

$$
x=\left(x_{j}\right)_{j \in J}, \quad y=\left(y_{j}\right)_{j \in J}, \quad f=\left(f_{j}\right)_{j \in J}, \quad g=\left(g_{j}\right)_{j \in J} .
$$

DEFINITION 3.1. The equilibrium of the model $\mathscr{E}$ with respect to a closed superlinear mapping $\mathscr{B}:\left(\mathbb{R}_{+}^{l}\right)^{J} \rightarrow\left(\mathbb{R}_{+}^{l}\right)^{J}$ with $\mathscr{B}(0)=\{0\}$ is a pair $(p, z)$, where $p=\left(\left(f_{j}, g_{j}\right)\right)_{j \in J}$ is a price vector and $z=\left(\left(x_{j}, y_{j}\right)\right)_{j \in J}$ is a state of the model, such that

(1) $f_{j} \neq 0$ and $x_{j}$ is a solution of the problem

$$
u_{j}\left(x_{j}\right) \rightarrow \max \quad \text { subject to } x_{j} \geq 0,\left[f_{j}, x_{j}\right] \leq \lambda_{j},
$$

for all $j \in J$;

(2) $y_{j}$ is a solution of the problem

$$
\left[g_{j}, y_{j}\right] \rightarrow \max \text { subject to } y_{j} \in Y_{j}
$$

for all $j \in J$;

(3) the four-tuple $(y, x, g, f)$ is matched with respect to the mapping $\mathscr{B}$.

We shall give three examples illustrating this definition.

EXAMPLE 1. Let $\mathscr{D}$ be a mapping of free exchange (see Definition 2.3). It is known (see [7], Lemma 8.17) that a four-tuple $(y, x, g, f)$ is matched with respect to mapping $\mathscr{D}$ if and only if there exists a vector $\bar{f}$ such that

$$
g_{j} \geq \tilde{f} \geq f_{j} \quad \text { for all } j
$$


and

$$
\left[g_{j}-\bar{f}, y_{j}\right]=\left[\bar{f}-f_{j}, x_{j}\right]=0 .
$$

Thus each agent would like to sell his or her products on at prices higher than $\bar{f}$ and to purchase at prices cheaper than $\bar{f}$. Nevertheless actual trades are made only at these prices. The exchange mapping $\mathscr{D}$ describes the classical exchange between agents and our definition leads to a version of classical equilibrium in this case. We shall give an alternative proof that Definition 3.1 leads to (2) and (3) in this paper (see Remark 4.2).

EXAMPLE 2. Consider the model $\mathscr{E}_{1}$ defined by the data:

$$
\begin{gathered}
I=J=\{1,2\}, \quad Y_{1}=Y_{2}=\{\bar{y}\}, \quad \text { with } \bar{y}=(1,1) ; \quad \lambda_{1}=\lambda_{2}=1, \\
u_{1}\left(x_{1}\right)=\left[\ell_{1}, x_{1}\right], \quad u_{2}\left(x_{2}\right)=\left[\ell_{2}, x_{2}\right],
\end{gathered}
$$

where $\ell_{j}=\left(\ell_{j}^{1}, \ell_{j}^{2}\right) \in \mathbb{R}_{+}^{2}, \ell_{j}^{1}+\ell_{j}^{2}=1, \ell_{j}^{1}>0, \ell_{j}^{2}>0, j=1,2$. Assume also that

$$
\mathscr{B}\left(y_{1}, y_{2}\right)=\left\{\left(x_{1}, x_{2}\right): 0 \leq x_{1} \leq y_{1}, 0 \leq x_{2} \leq y_{2}\right\} \text {. }
$$

It is easy to check that $\mathscr{B}^{*}\left(f_{1}, f_{2}\right)=\left\{\left(g_{1}, g_{2}\right): g_{j} \geq f_{j}, j=1,2\right\}$, so the four-tuple $\left(\left(y_{1}, y_{2}\right)\left(x_{1}, x_{2}\right),\left(g_{1}, g_{2}\right),\left(f_{1}, f_{2}\right)\right)$ is matched with respect to $\mathscr{B}$ if and only if

$$
\begin{gathered}
x_{1} \leq y_{1}, \quad x_{2} \leq y_{2}, \quad f_{1} \leq g_{1}, \quad f_{2} \leq g_{2} \\
{\left[f_{1}, x_{1}\right]+\left[f_{2}, x_{2}\right]=\left[g_{1}, y_{1}\right]+\left[g_{2}, y_{2}\right]}
\end{gathered}
$$

Assume the pair

$$
\left(\left(y_{1}, y_{2}\right),\left(x_{1}, x_{2}\right)\right),\left(\left(g_{1}, g_{2}\right),\left(f_{1}, f_{2}\right)\right)
$$

is an equilibrium of the model $\mathscr{E}_{1}$ with respect to the mapping $\mathscr{B}$. Clearly $y_{1}=y_{2}=\bar{y}$. By the definition of equilibrium the vector $x_{j}(j=1,2)$ is a solution of the problem

$$
\left[\ell_{j}, x\right] \rightarrow \max \quad \text { subject to }\left[f_{j}, x\right] \leq 1, x \geq 0
$$

and (4) holds.

We shall consider various possible cases for vectors $f_{1}, f_{2}$ to appear as equilibrium prices.

(1) $f_{1}=\ell_{1}, f_{2}=\ell_{2}$. Clearly an arbitrary nonnegative vector $x^{\prime}$ with the property $\left[\ell_{j}, x^{\prime}\right]=1$ is a solution of the problem (6). It easily follows from (4) that there exists a unique equilibrium in this case with $x_{j}=\bar{y}=(1,1)$ and $g_{j}=f_{j}=\ell_{j}$ for $j=1,2$.

(2) The vectors $f_{j}$ are proportional to vectors $\ell_{j}$, that is there are numbers $\mu_{j}>0$ such that $f_{j}=\mu_{j} \ell_{j}(j=1,2)$. It is easy to check that an equilibrium does not exist if at least one of the numbers $\mu_{j}$ is not equal to one. 
(3) The vector $f_{1}$ is not proportional to $\ell_{1}$ and both coordinates $f_{1}^{1}, f_{1}^{2}$ of this vector are positive; $f_{2}$ is an arbitrary positive vector. A solution $x_{1}$ of the problem (6) with $j=1$ is either the vector $\left(1 / f_{1}^{1}, 0\right)$ or the vector $\left(0,1 / f_{1}^{2}\right)$. Assume there exists an equilibrium (5). Since $y_{1} \geq x_{1}$ and $g_{1} \geq f_{1}$ and one of the coordinates of the vector $x_{1}$ is equal to zero, it follows that $\left[g_{1}, y_{1}\right]>\left[f_{1}, x_{1}\right]$. Also $\left[g_{2}, y_{2}\right] \geq\left[f_{2}, x_{2}\right]$, so the equality in (4) does not hold. Thus the equilibrium does not exist in this case. Symmetrically the equilibrium does not exist if $f_{2}$ is a vector with positive coordinates, not proportional to $\ell_{2}$.

(4) One of the coordinates of the vector $f_{j}$ with $j=1$ or $j=2$ is equal to zero. Clearly the problem (6) has no solution for the given $j$ so the equilibrium does not exist in this case either.

Thus there exists a unique equilibrium for the the model $\mathscr{E}_{1}$ with respect to the mapping $\mathscr{B}$ and the equilibrium prices are determined by utility functions.

EXAMPLE 3. Let $f_{1}, f_{2}$ be positive two-dimensional vectors. We shall denote by $S_{j}$ the simplex $\left\{x \geq 0:\left[f_{j}, x\right] \leq 1\right\}$. We now consider the model $\mathscr{E}_{2}$ with $1, J,\left(\lambda_{j}\right)_{j \in J}$ and $\left(u_{j}\right)_{j \in J}$ as in Example 2 and $Y_{j}=S_{j}$ for $j=1,2$. We shall study an equilibrium with respect to the same mapping $\mathscr{B}$ as in Example 2 assuming that vectors $f_{j}$ are not proportional to $\ell_{j}, j=1,2$. Clearly one of the coordinates of a solution $x_{j}$ of the problem (6) is equal to zero. Assume that $x_{j}=\left(1 / f_{j}{ }^{1}, 0\right)$. Let $y_{j}=x_{j}$ and $g_{j}=f_{j}$. Then

$$
\max _{y_{j}^{\prime} \in Y_{j}}\left[g_{j}, y_{j}^{\prime}\right]=\max \left\{\left[g_{j}, y_{j}^{\prime}\right]:\left[g_{j}, y_{j}^{\prime}\right] \leq 1\right\} .
$$

So the function $y^{\prime} \rightarrow\left[g_{j}, y^{\prime}\right]$ achieves its maximum over the set $Y_{j}$, in particular at the point $y_{j}$. Clearly the pair (5) with the given $y_{j}, x_{j}, f_{j}$ and $g_{j}$ forms an equilibrium of the model $\mathscr{E}_{2}$ with respect to the mapping $\mathscr{B}$. Thus the equilibrium prices are determined in this example by production sets $Y_{j}$.

\section{Equilibrium in the presence of public goods}

In this section we shall consider Definition 3.1 in order to define an equilibrium for models $\mathscr{E}$ with fixed budgets and superlinear connections in the presence of public goods. Suppose that there are both individual and public goods in the economy under consideration. Let us denote the set of individual goods by $I_{1}$ and the set of public goods by $I_{2}$ so $I=I_{1} \cup I_{2}$. The main property of individual and public goods is that without any restriction on free exchange it is possible to exchange a vector $y=\left(y_{j}\right)_{j J}$ for an arbitrary vector $x=\left(x_{j}\right)_{j \in J}$ such that

$$
\left(\forall i \in I_{1}\right) \quad \sum_{j} x_{j}^{i} \leq \sum_{j} y_{j}^{i} ; \quad\left(\forall i \in I_{2}\right) \quad x_{j}^{i} \leq \sum_{j} y_{j}^{i} .
$$


We assume that there are some restrictions on free exchange expressed in the form

$$
S x=T y,
$$

where $S, T:\left(\mathbb{R}_{+}^{I}\right)^{J} \rightarrow \mathbb{R}^{L}$ are linear nonnegative operators.

For simplicity we will denote the space of individual goods $\mathbb{R}^{I_{1}}$ by $\mathbb{R}^{i n}$ and the space of public goods $\mathbb{R}^{I_{2}}$ by $\mathbb{R}^{p u}$. For a vector $x \in \mathbb{R}^{I}$ we have the natural decomposition $x=x^{i n}+x^{p u}$ where $x^{i n}=\left(x^{i}\right)_{i \in I_{1}}$ is the 'individual' part of the vector $x$ and $x^{p u}=\left(x^{i}\right)_{i \in I_{2}}$ is the 'public' part of this vector.

Thus we can represent a superlinear mapping $\mathscr{G}$ which describes exchange with restrictions in the presence of public goods in the form

$$
\mathscr{G}(y)=\left\{x=\left(x_{j}\right)_{j \in J} \in\left(\mathbb{R}_{+}^{I}\right)^{J}: \sum_{j} x_{j}^{i n}=\sum_{j} y_{j}^{i n}, x_{j}^{p u} \leq \sum_{j} y_{j}^{p u}, S x=T y\right\},
$$

where $y=\left(y_{j}\right)_{j \in J} \in\left(\mathbb{R}_{+}^{I}\right)^{J}$. Assume the set $\mathscr{G}(y)$ is nonempty for all $y \in\left(\mathbb{R}_{+}^{I}\right)^{J}$. Clearly if $x \in \mathscr{G}(y)$ then there are the usual balance equations for individual goods and each participant can consume all the available quantities of public goods; at the same time the exchange activity of agents is restricted by the equality $S x=T y$.

In order to apply Definition 3.1 we need a description of matched four-tuples with respect to the the mapping $\mathscr{G}$.

First we will describe the conjugate mapping $\mathscr{G}^{*}$.

PROPOSITION 4.1. Let $f=\left(f_{j}\right)_{j \in J} \in\left(\mathbb{R}_{+}^{l}\right)^{J}$. Then $g=\left(g_{j}\right)_{J \in J} \in \mathscr{G}^{*}(f)$ if and only if there exist vectors $\ell \in \mathbb{R}_{+}^{i n} ; \lambda \in \mathbb{R}^{L}$ and $h=\left(h_{j}\right)_{j \in J} \in\left(\mathbb{R}^{p u}\right)^{J}, h_{j} \geq 0$ such that for all $j \in J$

$$
\begin{array}{ll}
\ell+\left(S^{*} \lambda\right)_{j}^{i n} \geq f_{j}^{i n} ; & \left(S^{*} \lambda\right)_{j}^{p u}+h_{j} \geq f_{j}^{p u} \\
g_{j}^{i n} \geq \ell+\left(T^{*} \lambda\right)_{j}^{i n} ; & g_{j}^{p u} \geq\left(T^{*} \lambda\right)_{j}^{p u}+\bar{h}
\end{array}
$$

with $\bar{h}=\sum_{k} h_{k}$

PROOF. For $f=\left(f_{j}\right)_{j \in J} \in\left(\mathbb{R}_{+}^{I}\right)^{J}$, set

$$
s_{f}(y)=\max \{[f, x]: x \in \mathscr{G}(y)\} \quad \forall y \in\left(\mathbb{R}_{+}^{I}\right)^{J} .
$$

The functional $s_{f}$, defined on the cone $\left(\mathbb{R}_{+}^{l}\right)^{J}$ by $(12)$, is superlinear. Clearly the set $\mathscr{G}^{*}(f)$ coincides with the superdifferential

$$
\partial s_{f}=\left\{g:[g, y] \geq s_{f}(y) \quad \forall y \in\left(\mathbb{R}_{+}^{l}\right)^{J}\right\}
$$

of the functional $s_{f}$. We shall use the notation: $x^{\Delta}=(x, \ldots, x) \in\left(\mathbb{R}_{+}^{I}\right)^{J}$, where $x \in \mathbb{R}_{+}^{I}$. 
We now represent $s_{f}(y)$ as the optimal value of the linear programming problem:

$$
[f, x] \rightarrow \max \quad \text { subject to } P_{1} x=b_{1}, P_{2} x \leq b_{2} ; x \geq 0
$$

where

$$
b_{1}=\left(\sum_{j} y_{j}^{i n}, T y\right) \in \mathbb{R}^{i n} \times \mathbb{R}^{L}, \quad b_{2}=\left(\sum_{j} y_{j}^{p u}\right)^{\Delta} \in\left(\mathbb{R}^{p u}\right)^{J}
$$

and $P_{1}:\left(\mathbb{R}_{+}^{l}\right)^{J} \rightarrow \mathbb{R}^{i n} \times \mathbb{R}^{L}, P_{2}:\left(\mathbb{R}_{+}^{I}\right)^{J} \rightarrow\left(\mathbb{R}^{p u}\right)^{J}$ are linear operators defined by the formulae

$$
P_{1} x=\left(\sum_{j} x_{j}^{i n}, S x\right), \quad P_{2} x=\left(x^{p u}\right)^{\Delta} .
$$

The dual problem of (13) is

$$
\left[H_{1}, b_{1}\right]+\left[H_{2}, b_{2}\right] \rightarrow \min \quad \text { subject to } P_{1}^{*} H_{1}+P_{2}^{*} H_{2} \geq f, H_{2} \geq 0 .
$$

(We denote by $A^{*}$ the conjugate operator to a linear operator $A$.) Let us describe vectors $H_{1}, H_{2}$ such that $P_{1}^{*} H_{1}+P_{2}^{*} H_{2} \geq f$. For $H_{1}=(\ell, \lambda) \in \mathbb{R}^{i n} \times \mathbb{R}^{L}$ and $H_{2}=\left(h_{j}\right)_{j \in J} \in\left(\mathbb{R}_{+}^{I}\right)^{J}$ we have

$$
\begin{aligned}
{\left[P_{1}^{*} H_{1}+P_{2}^{*} H_{2}, x\right] } & =\left[H_{1}, P_{1} x\right]+\left[H_{2}, P_{2} x\right] \\
& =\left[\ell, \sum_{j} x_{j}^{i n}\right]+[\lambda, S x]+\left[H_{2}, x^{p u}\right] \\
& =\left[\ell^{\Delta}, x^{i n}\right]+\left[\left(S^{*} \lambda\right)^{i n}, x^{i n}\right]+\left[\left(S^{*} \lambda\right)^{p u}, x^{p u}\right]+\left[H_{2}, x^{p u}\right] .
\end{aligned}
$$

So

$$
\left[P_{1}^{*} H_{1}+P_{2}^{*} H_{2}-f, x\right]=\left[\ell^{\Delta}+\left(S^{*} \lambda\right)^{i n}, x^{i n}\right]+\left[\left(S^{*}\right)^{p u}+H_{2}, x^{p u}\right] .
$$

Since $P_{1}^{*} H_{1}+P_{2}^{*} H_{2} \geq f$ if and only if $\left[P_{1}^{*} H_{1}+P_{2}^{*} H_{2}-f, x\right] \geq 0$ for all $x \geq 0$ it follows that the set $\left\{\left(H_{1}=(\ell, \lambda), H_{2}\right): P_{1}^{*} H_{1}+P_{2}^{*} H_{2} \geq f\right\}$ coincides with the set $U(f)$ :

$$
U(f)=\left\{\left(\ell, \lambda, H_{2}\right): H_{2} \geq 0 ; \ell^{\Delta}+\left(S^{*} \lambda\right)^{i n} \geq f^{i n} ;\left(S^{*} \lambda\right)^{p u}+H_{2} \geq f^{p u}\right\} .
$$

Thus

$$
\begin{aligned}
s_{f}(y) & =\max _{\substack{x \geq 0, P_{1} x=b_{1}, P_{2} x \leq b_{2}}}[f, x]=\min _{\substack{\left(H_{1}, H_{2}\right): H_{2} \geq 0, P_{1}^{*} H_{1}+P_{2}^{*} \geq f}}\left[H_{1}, b_{1}\right]+\left[H_{2}, b_{2}\right] \\
& =\min _{\left(\ell, \lambda, H_{2}\right) \in U(f)}\left[\ell, \sum_{j} y_{j}^{i n}\right]+[\lambda, T y]+\sum_{k}\left[h_{k}, \sum_{j} y_{j}^{p u}\right] \\
& =\min _{\left(\ell, \lambda, H_{2}\right) \in U(f)} \sum_{j}\left[\ell+\left(T^{*} \lambda\right)_{j}^{i n}, y_{j}^{i n}\right]+\sum_{j}\left[\left(T^{*} \lambda\right)_{j}^{p u}+\bar{h}, y_{j}^{p u}\right] \\
& =\min _{\left(\ell, \lambda, H_{2}\right) \in U(f)}\left[\left(\ell^{\Delta}+\left(T^{*} \lambda\right)^{i n}, y^{i n}\right]+\left[\left(T^{*}(\lambda)\right)^{p u}+\bar{h}^{\Delta}, y^{p u}\right],\right.
\end{aligned}
$$


with $\bar{h}=\sum_{k} h_{k}$. For $H_{1}=(\ell, \lambda)$ and $H_{2}=\left(h_{j}\right)_{j \in J}$, set

$$
B\left(H_{1}, H_{2}\right)=\left(\ell^{\Delta}+\left(T^{*} \lambda\right)^{i n},\left(T^{*}(\lambda)\right)^{p u}+\bar{h}^{\Delta}\right) .
$$

Clearly $B$ is a linear operator. Thus

$$
s_{f}(y)=\min _{H_{1}=(\ell, \lambda), H_{2} \in U(f)} B\left(H_{1}, H_{2}\right)(y)=\min _{g \in B(U(f))}[g, y]
$$

Since $U(f)$ is a polyhedral convex set and $B$ is a linear operator it follows that the set $B(U(f))$ is also convex and polyhedral. It is easy to check, by applying properties of superlinear functions defined on a closed convex cone (see, for example, [7]), that $\partial s_{f}=B(U(f))+\left(\mathbb{R}_{+}^{I}\right)^{J}$. Thus $g \in \mathscr{G}^{*}(f)=\partial s_{f}$ if and only if there exists a vector $\left(\ell, \lambda,\left(h_{j}\right)\right) \in U(f)$, that is, $h_{j} \geq 0$ and

$$
\ell+\left(S^{*} \lambda\right)_{j}^{i n} \geq f_{j}^{i n} ; \quad\left(S^{*} \lambda\right)_{j}^{p u}+h_{j} \geq f_{j}^{p u} \quad \forall j \in J
$$

with

$$
g_{j}^{i n} \geq \ell+\left(T^{*} \lambda\right)_{j}^{i n} ; \quad g_{j}^{p u} \geq\left(T^{*} \lambda\right)_{j}^{p u}+\vec{h} \quad \forall j \in J
$$

PROPOSITION 4.2. A four-tuple $(y, x, g, f)$ with

$$
y=\left(y_{j}\right)_{j \in J}, \quad x=\left(x_{j}\right)_{j \in J}, \quad g=\left(g_{j}\right)_{j \in}, \quad f=\left(f_{j}\right)_{j \in J}
$$

is matched with respect to $\mathscr{G}$ if and only if $x \in \mathscr{G}(y)$ and there exist vectors $\ell \in \mathbb{R}^{\text {in }}$, $\lambda \in \mathbb{R}^{L}$ and $h \in\left(\mathbb{R}^{p u}\right)^{J}, h \geq 0$, such that (10) and (11) hold for all $j \in J$ and

$$
\begin{gathered}
{\left[f^{i n}-\left(\ell^{*}+\left(S^{*} \lambda\right)^{i n}\right), x^{i n}\right]=0, \quad\left[f^{p u}-\left(\left(S^{*} \lambda\right)^{p u}+h\right), x^{p u}\right]=0} \\
{\left[h, x^{p u}-\left(\sum_{k \in J} y_{k}\right)^{\Delta}\right]=0} \\
{\left[\ell^{\Delta}+T^{*} \lambda, y^{i n}\right]=\left[g, y^{i n}\right], \quad\left[T^{*} \lambda+\bar{h}^{\Delta}, y^{p u}\right]=\left[g, y^{p u}\right] .}
\end{gathered}
$$

Proof. Let $(y, x, g, \dot{f})$ be a four-tuple such that $x \in \mathscr{G}(y)$ and $g \in \mathscr{G}^{*}(f)$. Then (7) and (8) are valid and there is a vector $\left(\ell, \lambda,\left(h_{j}\right)\right)_{j \in J}$ such that (10) and (11) hold. Since $g \in \mathscr{G}^{*}(f)$ it follows that $[f, x] \leq[g, y]$. It is easy to check that this nonstrict inequality becomes an equality if and only if (15) to (17) hold.

REMARK 4.1. The equalities (15)-(17) represent complementary slackness conditions. For example, consider equality (16). This equality is clearly equivalent to the system:

$$
\sum_{i \in I_{2}} h_{j}^{i}\left(x_{j}^{i}-\bar{y}^{i}\right)=0 \quad(j \in J)
$$


with $\bar{y}=\sum_{j \in J} y_{j}$. It follows from the definition of the mapping $\mathscr{G}$ and (18) that for all $j \in J$ and $i \in I_{2}$ we have

$$
h_{j}^{i}\left(x_{j}^{i}-\bar{y}^{i}\right)=0
$$

These results allow us to define an equilibrium for the model under consideration. We shall exploit Definition 3.1 and Proposition 5.2.

DEFINITION 4.1. The equilibrium of the model $\mathscr{E}$ with superlinear connection $S x=$ $T y$, individual goods $i \in I_{1}$ and public goods $i \in I_{2}$, is the pair $(p, z)$, where $p=\left(\left(f_{j}, g_{j}\right)\right)_{j \in J}$ is a price vector and $z=\left(\left(x_{j}, y_{j}\right)\right)_{j \in J}$ is a state of the model, such that

(1) $f_{j} \neq 0$ and $x_{j}$ is a solution of the problem

$$
u_{j}\left(x_{j}\right) \rightarrow \max \quad \text { subject to } x_{j} \geq 0,\left[f_{j}, x_{j}\right] \leq \lambda_{j}
$$

for all $j \in J$;

(2) $y_{j}(j \in J)$ is a solution of the problem

$$
\left[g_{j}, y_{j}\right] \rightarrow \max \quad \text { subject to } y_{j} \in Y_{j}
$$

(3) inequalities (10) and (11) and equalities (15)-(17) hold for all $j \in J$.

REMARK 4.2. If the set $I_{2}$ is empty then we obtain a definition of equilibrium with individual goods and linear restriction on exchange. If in addition there is no restriction, that is, $S=T=0$, we obtain a version of the classical definition of equilibria given in Example 1.

If $S=T=0$ and $I_{2} \neq \emptyset$ then the definition transforms to a version of the classical definition of the equilibrium with public goods (see, for example, [2]). Since $S=T=0$ it follows that the inequalities (10) and (11) and the equalities (15)-(17) lead to the following (with respect to public goods): for all $j \in J$ there exists a price vector $h_{j} \geq 0$ such that

$$
\begin{gathered}
h_{j} \geq f_{j}^{p u}, \quad g_{j}^{p u} \geq \bar{h}, \quad \text { where } \quad \bar{h}=\sum_{j \in J} h_{j}, \\
{\left[h_{j}-f_{j}^{p u}, x_{j}^{p u}\right]=\left[g_{j}^{p u}-\bar{h}, y_{j}^{p u}\right]=0, \quad\left[h_{j}, x_{j}^{p u}-\sum_{k \in J} y_{k}\right]=0 .}
\end{gathered}
$$

Thus we can consider $\bar{h}$ as a total price for public goods and $h_{j}$ as the $j$ th agent's contribution to this price. 


\section{Extremality and existence of equilibria}

Let us consider the model $\mathscr{E}$ defined by the formula (1):

$$
\mathscr{E}=\left\{I, J,\left(Y_{j}\right)_{j \in J},\left(u_{j}\right)_{j \in J},\left(\lambda_{j}\right)_{j \in J}\right\} .
$$

In order to describe equilibria for this model we need the extremal problem

$$
\sum_{j \in J} \lambda_{j} \log u_{j}\left(x_{j}\right) \rightarrow \max \quad \text { subject to } x=\left(x_{j}\right)_{j \in J} \in \mathscr{B}(y), y \in Y,
$$

where $Y=\prod_{j \in J} Y_{j}$. We assume as usual that $\log 0=-\infty$.

We now show that under some additional natural assumptions a solution of problem (19) can be considered as the consumption part of an equilibrium of the model $\mathscr{E}$.

\section{THEOREM 5.1. Assume that}

(1) $\mathscr{B}$ is a polyhedral mapping;

(2) the set $K=\cup\{x \in \mathscr{B}(y): y \in Y\}$ contains a strictly positive vector.

Then for each solution $\bar{x}$ of the problem (19) there exist vectors $\bar{y}, \bar{f}, \bar{g}$ such that the pair $((\bar{y}, \bar{x}),(\bar{f}, \bar{g}))$ forms an equilibrium of the model $\mathscr{E}$.

Proof. Let us consider the function $q(x)=\sum_{j \in J} \lambda_{j} \log u_{j}\left(x_{j}\right)$ defined on the cone $\left(\mathbb{R}_{+}^{I}\right)^{J}$. Clearly $q$ is a concave function. We shall denote by $\partial q(x)$ the superdifferential of the function $q$ at a point $x$. Since $Y$ is a compact convex set and $\mathscr{B}$ is a superlinear mapping it follows that the set $K=\mathscr{B}(Y)$ is compact and convex. Let $\bar{x}=\left(\bar{x}_{j}\right)_{j \in J}$ be a solution of the problem (19). Since the set $K$ contains a strictly positive vector it follows that $q(\bar{x})>-\infty$ so $u_{j}\left(\bar{x}_{j}\right)>0$ for all $j \in J$. It follows from well-known necessary conditions for a maximum of a concave function over a convex set that there exists a vector $\bar{f} \in \partial q(\bar{x})$ such that

$$
\bar{f}(\bar{x})=\max \{\bar{f}(x): x \in K\} .
$$

Let us represent $\bar{f}$ in terms of superdifferentials of the functions $u_{j}$. For this purpose we shall express $q$ in the form $q(x)=\phi\left(u_{1}\left(x_{1}\right), \ldots, u_{m}\left(x_{m}\right)\right)$ where $\phi(z)=$ $\sum_{j \in J} \lambda_{j} \log \left(z_{j}\right)$. Since the function $\phi$ is a concave increasing function we can apply well-known results from convex analysis (see, for example, [3], Lemma 12.2) which show that $\bar{f} \in \partial q(\bar{x})$ if and only if $\bar{f}(x)=\sum_{j \in J} \mu_{j} \ell_{j}\left(x_{j}\right)$ where $\mu=\left(\mu_{j}\right) \in \partial \phi(z)$ with $z=\left(u_{1}\left(\bar{x}_{1}\right), \ldots, u_{m}\left(\bar{x}_{m}\right)\right)$ and $\ell_{j} \in \partial u_{j}\left(\bar{x}_{j}\right)$. Since $\phi(z)$ is a smooth function we have (assuming that $z_{j}>0$ for all $j \in J$ )

$$
\partial \phi(z)=\left\{\left(\frac{\partial \phi}{\partial z_{1}}, \ldots \frac{\partial \phi}{\partial z_{m}}\right)\right\}=\left\{\left(\frac{\lambda_{1}}{z_{1}}, \ldots, \frac{\lambda_{m}}{z_{m}}\right)\right\} .
$$


Thus $\bar{f} \in \partial q(\bar{x})$ if and only if

$$
\bar{f}=\left(\frac{\lambda_{1}}{u_{1}\left(\bar{x}_{1}\right)} \ell_{1}, \ldots, \frac{\lambda_{m}}{u_{m}(\bar{x})} \ell_{m}\right),
$$

where $\ell_{j} \in \partial u_{j}\left(\bar{x}_{j}\right)$ for all $j \in J$.

Thus for the solution $\bar{x}$ of the problem (19) there exists a vector $\bar{f}$ of the form (21) such that the equality $(20)$ holds.

We shall denote the vector $\left(\lambda_{j} / u_{j}\left(\bar{x}_{j}\right)\right) \ell_{j}$ by $\bar{f}_{j}$. Since $u_{j}$ is a superlinear function on $\mathbb{R}_{+}^{\prime}$ it follows that $\ell_{j} \neq 0$, therefore $\bar{f}_{j} \neq 0$. We now check that the vector $\bar{x}_{j}$ is a solution of the problem

$$
u_{j}\left(x_{j}\right) \rightarrow \max \quad \text { subject to } x_{j} \geq 0,\left[\overline{f_{j}}, x_{j}\right] \leq \lambda_{j} .
$$

Indeed if $x_{j} \in \mathbb{R}_{+}^{I}$ and $\left[\overrightarrow{f_{j}}, x_{j}\right] \leq \lambda_{j}$ then $\left[\ell_{j}, x_{j}\right] \leq u_{j}\left(\bar{x}_{j}\right)$. Also $\ell_{j} \in \partial u\left(\bar{x}_{j}\right)$ implies $u_{j}\left(x_{j}\right) \leq\left[\ell_{j}, x_{j}\right]$ for all $x_{j} \geq 0$. Thus $u_{j}\left(x_{j}\right) \leq u_{j}\left(\bar{x}_{j}\right)$.

We now find vectors $\bar{g}$ and $\bar{y}$ that together with $\bar{f}$ and $\bar{x}$ form an equilibrium. Recall that $\mathscr{B}$ is a polyhedral mapping. By applying (20), a standard minimax theorem and Theorem 2.1 (for polyhedral mappings), we can conclude that

$$
[\bar{f}, \bar{x}]=\max _{y \in Y} \max _{x \in \mathscr{B}(y)}[\bar{f}, x]=\max _{y \in Y} \min _{g \in \mathscr{B}^{*}(\bar{f})}[g, y]=\min _{g \in \mathscr{B} *(\bar{f})} \max _{y \in Y}[g, y] .
$$

Let vectors $\bar{g} \in \mathscr{B}^{*}(\bar{f})$ and $\bar{y} \in Y$ be defined by the equalities

$$
\begin{aligned}
\min _{g \in \mathscr{B}^{*}(\bar{f})} \max _{y \in Y}[g, y] & =\max _{y \in Y}[\bar{g}, y], \\
\max _{y \in Y}[\bar{g}, y] & =[\bar{g}, \bar{y}] .
\end{aligned}
$$

Then $[\bar{f}, \bar{x}]=[\bar{g}, \bar{y}]$, that is, the four-tuple $(\bar{y}, \bar{x}, \bar{g}, \bar{f})$ is matched with respect to $\mathscr{B}$. Also the equality (23) shows that

$$
\max _{y_{j} \in Y_{j}}\left[\bar{g}_{j}, y_{j}\right]=\left[\bar{g}_{j}, \bar{y}_{j}\right]
$$

for all $j \in J$. It follows from (22) and (24) that the pair $((\bar{y}, \bar{x}),(\bar{g}, \bar{f}))$ forms an equilibrium of the model $\mathscr{E}$.

COROLLARY 5.1. If conditions (1) and (2) of Theorem 5.1 hold, then the model $\mathscr{E}$ has an equilibrium with respect to mapping $\mathscr{B}$.

Indeed the concave upper semicontinuous function $q$ achieves its maximum over the compact set $K$. By applying Theorem 5.1 we can deduce that the equilibrium exists.

We now shall prove the inverse theorem. The consumption part of an arbitrary equilibrium of the model $\mathscr{E}$ is a solution of the problem (19) without any additional assumptions. At first we shall prove the following simple assertion. 
LEMMA 5.1. Let $\ell, f \in \mathbb{R}_{+}^{\prime}$ and $\bar{x}$ be a solution of the problem

$$
[\ell, x] \rightarrow \max \quad \text { subject to }[f, x] \leq \lambda, x \geq 0 .
$$

Further assume that the value of this problem is finite. Let

$$
I_{f}=\left\{i \in I: f^{i}>0\right\}, \quad I_{\ell}=\left\{i \in I: \ell^{i}>0\right\}, \quad I_{\bar{x}}=\left\{i \in I: \bar{x}_{i}>0\right\} .
$$

Then $I_{\ell} \subseteq I_{f}, \lambda \ell_{i} \leq \beta f_{i}$ for all $i \in I_{f}$ and $\lambda \ell_{i}=\beta f_{i}$ for all $i \in I_{\bar{x}} \cap I_{\ell}$ where $\beta$ is the value of the problem (25).

PROOF. Since the value of the problem (25) is finite it follows that $I_{\ell} \subseteq I_{f}$. It is easy to check that the value $\beta$ of the problem (25) is equal to $\lambda \max _{i \in I_{f}}\left(\ell_{i} / f_{i}\right)$. Thus $\lambda \ell_{i} \leq \beta f_{i}$ for all $i \in I_{f}$. Let $\alpha_{i}=f_{i} \bar{x}_{i} / \lambda$. Then $\sum_{i \in I} \alpha_{i}=[f, \bar{x}] / \lambda=1$ and $\alpha_{i} \geq 0$. Clearly $\alpha_{i}>0$ for $i \in I_{f} \cap I_{\bar{x}}$. We have

$$
\beta=\max _{i \in I_{f}} \frac{\lambda \ell_{i}}{f_{i}}=[\ell, \bar{x}]=\sum_{i \in I} \alpha_{i} \frac{\lambda \ell_{i}}{f_{i}} .
$$

So $\lambda \ell_{i}=\beta f_{i}$ for all $i \in I_{\ell}$ with $\alpha_{i}>0$. Thus $\lambda \ell_{i}=\beta f_{i}$ for $i \in I_{\bar{x}} \cap I_{\ell}$.

THEOREM 5.2. Let a pair $((\bar{y}, \bar{x}),(\bar{g}, \bar{f}))$ be an equilibrium of a model $\mathscr{E}$ with respect to a mapping $\mathscr{B}$. Then the vector $\bar{x}$ is a solution of the problem (19).

PROOF. First we calculate the maximum of the equilibrium prices $\bar{f}$ over the set $K=\mathscr{B}(Y)$. We have

$$
\max _{x \in K}[\bar{f}, x]=\max _{y \in Y} \max _{x \in \mathscr{B}(y)}[\bar{f}, x]=\max _{y \in Y} \min _{g \in \mathscr{B}^{*}(\bar{f})}[g, y] \leq \max _{y \in Y}[\bar{g}, y]=[\bar{g}, \bar{y}]=[\bar{f}, \bar{x}] .
$$

Also

$$
\max _{y \in Y} \max _{x \in \mathscr{B}(y)}[\bar{f}, x] \geq[\bar{f}, \bar{x}]
$$

Thus

$$
\max _{x \in K}[\bar{f}, x]=[\bar{f}, \bar{x}]
$$

We now consider the following extremal problems for all $j \in J$ :

$$
u_{j}(x) \rightarrow \max \quad \text { subject to }\left[\overline{f_{j}}, x_{j}\right] \leq \lambda_{j}, x_{j} \geq 0 .
$$

Since $\bar{x}_{j}$ is a solution of the problem (28) it follows that there exists a vector $\ell_{j} \epsilon$ $\partial u_{j}\left(\bar{x}_{j}\right)$ such that $\bar{x}_{j}$ is also a solution of the problem

$$
\left[\ell_{j}, x_{j}\right] \rightarrow \max \quad \text { subject to }\left[\overline{f_{j}}, x_{j}\right] \leq \lambda_{j}, x_{j} \geq 0 .
$$


It follows from Lemma 5.1 that

$$
\beta_{j} \overline{f_{j}^{i}} \geq \lambda_{j} \ell_{j}^{i} \quad\left(\forall i \in I_{\bar{f}_{j}}\right), \quad \beta_{j} \bar{f}_{j}^{i}=\lambda_{j} \ell_{j}^{i} \quad\left(\forall i \in I_{\ell_{j}} \cap I_{\bar{x}_{j}}\right),
$$

where $I_{\overline{f_{j}}}, I_{\ell_{j}}$ and $I_{\bar{x}_{j}}$ are defined as in (26) and $\beta_{j}$ is the value of the problem (29). Since the set $\left\{x_{j} \geq 0:\left[f_{j}, x_{j}\right] \leq \lambda_{j}\right\}$ contains an interior point of the cone $\mathbb{R}_{+}^{n}$ and $u_{j}$ is positive on the interior of this cone it follows that $\beta_{j}>0$. The first relation in (30) shows that

$$
\max _{x \in K} \sum_{j \in J} \frac{\lambda_{j}}{\beta_{j}}\left[\ell_{j}, x_{j}\right] \leq \max _{x \in K} \sum_{j \in J}\left[\overline{f_{j}}, x_{j}\right]=\max _{x \in K}[\bar{f}, x] .
$$

Since $I_{\overline{f_{j}}} \subseteq I_{\ell_{j}}$ it follows that the second relation in (30) implies

$$
[\bar{f}, \bar{x}]=\sum_{j \in J}\left[\overline{f_{j}}, \bar{x}_{j}\right]=\sum_{j \in J} \frac{\lambda_{j}}{\beta_{j}}\left[\ell_{j}, \bar{x}_{j}\right] .
$$

Let $\ell=\left(\left(\lambda_{j} / \beta_{j}\right) \ell_{j}\right)_{j \in J}$. By combining (27), (31) and (32) we deduce that

$$
\max _{x \in K}[\ell, x]=[\ell, \bar{x}] .
$$

We now check that $\ell \in \partial q(\bar{x})$ where $q(x)=\sum_{j \in J} \lambda_{j} \log u_{j}\left(x_{j}\right)$. Since $\ell_{j} \in \partial u_{j}(x)$ and $u_{j}$ is a superlinear function it follows that $u_{j}\left(\bar{x}_{j}\right)=\left[\ell_{j}, \bar{x}_{j}\right]=\beta_{j}$. So

$$
\ell=\left(\frac{\lambda_{1}}{u_{1}\left(\bar{x}_{1}\right)} \ell_{1}, \ldots, \frac{\lambda_{m}}{u_{m}\left(\bar{x}_{m}\right)} \ell_{m}\right) .
$$

This equality shows (see the proof of Theorem 5.1) that $\ell \in \partial q(\bar{x})$. Applying this fact in combination with (33) we deduce that $u(x) \leq[\ell, x] \leq[\ell, \bar{x}]=u(\bar{x})$ for all $x \in K$.

COROLlaRY 5.2. Let $X$ be the set of all $x \in\left(\mathbb{R}_{+}^{I}\right)^{J}$ with the property that there exist vectors $y, g, f$ such that the pair $((y, x),(g, f))$ is an equilibrium of the model $\mathscr{E}$. Then $X$ is a compact convex set.

COROllary 5.3. Let $x, x^{\prime} \in X$ where the set $X$ is defined in Corollary 5.2. Then $u_{j}\left(x_{j}\right)=u_{j}\left(x_{j}^{\prime}\right)$ for all $j$.

Proof. Assume there is an index $k$ such that $u_{k}\left(x_{k}\right) \neq u_{j}\left(x_{k}^{\prime}\right)$. Since the log function is a strictly concave function we have for $\alpha \in(0,1)$ that

$$
\begin{aligned}
\sum_{j} \lambda_{j} \log u_{j}\left(\alpha x_{j}+(1-\alpha) x_{j}^{\prime}\right) & \geq \sum_{j} \lambda_{j} \log \left(\alpha u_{j}\left(x_{j}\right)+(1-\alpha) u_{j}\left(x_{j}^{\prime}\right)\right. \\
& >\sum_{j} \lambda_{j} \alpha \log u_{j}\left(x_{j}\right)+\sum_{j}\left(1-\alpha_{j}\right) u_{j}\left(x_{j}^{\prime}\right) \\
& =A,
\end{aligned}
$$

where $A$ is the value of the problem (19). Thus we obtain a contradiction. 


\section{Differential properties of equilibria}

We shall consider in this section a family of equilibrium models

$$
\mathscr{E}_{\Lambda}=\left\{I, J,\left(Y_{j}\right)_{j \in J},\left(u_{j}\right)_{j \in J},\left(\lambda_{j}\right)_{j \in J}\right\}
$$

depending on the parameter $\Lambda=\left(\lambda_{j}\right)_{j \in J}$. We shall consider only strictly positive values of the parameter $\Lambda$ and study equilibria of the models $\mathscr{E}_{\Lambda}$ with respect to a polyhedral superlinear mapping $\mathscr{B}$ independent of $\Lambda$. Let us denote by $x_{j}(\Lambda)$ an equilibrium consumption of the agent $j$ for the model $\mathscr{E}_{\Lambda}$. Then we can apply Theorem 5.2 which shows that the vector $x(\Lambda)=\left(x_{j}(\Lambda)\right)_{j \in J}$ is a solution of the problem (19). We shall use the notation

$$
u_{j}(\Lambda)=u_{j}\left(x_{j}(\Lambda)\right) .
$$

It follows from Corollary 5.3 that the numbers $u_{j}\left(x_{j}(\Lambda)\right)$ depend only on the model $\mathscr{E}$ and the mapping $\mathscr{B}$ and coincide for all equilibria, hence the notation is appropriate.

We shall consider in this section the function

$$
\varphi(\Lambda)=\max _{x \in K} \sum_{j \in J} \lambda_{j} \log u_{j}\left(x_{j}\right)=\sum_{j \in J} \lambda_{j} \log u_{j}(\Lambda),
$$

where $K=\mathscr{B}(Y), Y=\prod_{j \in J} Y_{j}$.

PROPOSITION 6.1. The function $\varphi$ is continuously differentiable and

$$
\nabla \varphi(\Lambda)=\left(\log u_{j}(\Lambda)\right)_{j \in J},
$$

where $\left(u_{j}(\Lambda)\right)$ is defined by $(34)$.

PROOF. First we check that the function $\varphi$ is directionally differentiable, that is, for all $\mu$ there exists a directional derivative $\varphi^{\prime}(\Lambda, \mu)=\lim _{t \rightarrow+0}(1 / t)(\varphi(\Lambda+t \mu)-\varphi(\Lambda))$. Since $\varphi$ is a maximum of linear functions (with respect to $\Lambda$ ) we can apply well-known results about directional differentiability of a maximum function (see, for example, [3]) which show that the directional derivative $\varphi^{\prime}(\Lambda, \mu)$ does exist and

$$
\varphi^{\prime}(\Lambda, \mu)=\max _{x(\Lambda)=\left(x_{j}(\Lambda)\right)_{j \in J} \in R(\Lambda)} \sum_{j \in J} \mu_{j} \log u_{j}\left(x_{j}(\Lambda)\right),
$$

where $R(\Lambda)$ consists of all vectors $x(\Lambda)$ which maximize the function $(\Lambda, x) \rightarrow$ $\sum_{j \in J} \lambda_{j} u_{j}\left(x_{j}\right)$ over the set $K$. It follows from Corollary 5.3 that all solutions of this maximization problem have the same value $u_{j}\left(x_{j}(\Lambda)\right)=u_{j}(\Lambda)$. So

$$
\varphi^{\prime}(\Lambda, \mu)=\sum_{j \in J} \mu_{j} \log u_{j}(\Lambda)
$$


The function $\varphi$ is convex (more precisely, sublinear) as the maximum of linear functions $\Lambda \rightarrow \sum_{j \in J} \lambda_{j} u_{j}\left(x_{j}\right)$. It is well known (see, for example, [10, Theorems 25.2 and 25.4]) that linearity of the directional derivative $\varphi^{\prime}(\Lambda, \mu)$ of a convex function with respect to $\mu$ implies continuous differentiability of this function. If $\mu=\left(\mu_{k}\right)_{k \in J}$ with $\mu_{j}=1$ and $\mu_{k}=0$ for $k \neq j$ then we have

$$
\frac{\partial \varphi}{\partial \lambda_{j}}=\log u_{j}(\Lambda)
$$

Thus $\nabla \varphi(\Lambda)=\left(\log u_{j}(\Lambda)\right)_{j \in J}$.

COROLLARY 6.1. The equilibrium utility $u_{j}(\Lambda)$ is a continuous function of the budgets $\Lambda$.

COROLLARY 6.2. The function $\varphi$ satisfies the differential equality

$$
\varphi(\Lambda)=[\Lambda, \nabla \varphi(\Lambda)]
$$

PROPOSITION 6.2. Assume that the functions $u_{j}(\Lambda)$ defined by (34) are differentiable for all $j \in J$. Then these functions satisfy the system of differential equations given by

$$
\sum_{j \in J} \frac{\lambda_{j}}{u_{j}(\Lambda)} \frac{\partial}{\partial \lambda_{k}} u_{j}(\Lambda)=0 \quad(k \in J) .
$$

Proof. This follows easily by applying Proposition 6.1 and the fact that $\varphi(\Lambda)=$ $\sum_{j \in J} \lambda_{j} \log u_{j}(\Lambda)$

REMARK 6.1. Note that the system (36) does not depend on the production sets $Y_{j}$ nor on the restriction of exchange mapping $\mathscr{B}$.

We can express the system (36) in terms of rates of growth.

DEFINITION 6.1. The number $\gamma_{j}$ is called the rate of growth of the agent $j$ in a model $\mathscr{E}=\left\{I, J,\left(Y_{j}\right)_{j \in J},\left(u_{j}\right)_{j \in J},\left(\lambda_{j}\right)_{j \in J}\right\}$ if there exists an equilibrium $((\bar{y}, \bar{x}),(\bar{g}, \bar{f}))$ of this model such that

$$
\gamma_{j}=\frac{u_{j}\left(\bar{x}_{j}\right)}{\left[\bar{f}_{j}, \bar{x}_{j}\right]} .
$$

Rates of growth of models with fixed budgets play an important role in applications of these models to economic dynamics (see [7]). 
We have

Thus

$$
\max _{\substack{x_{j} \geq 0 \\\left[\overline{\left.f_{j}, x_{j}\right] \leq \lambda_{j}}\right.}} u_{j}\left(x_{j}\right)=\lambda \max _{\substack{x_{j} \geq 0 \\\left[\overline{f_{j}, x_{j}}\right] \leq 1}} u_{j}\left(x_{j}\right)=\lambda_{j} \frac{u_{j}\left(\bar{x}_{j}\right)}{\left[\overline{f_{j}}, \overline{x_{j}}\right]}=\lambda_{j} \gamma_{j}
$$

$$
u_{j}\left(\bar{x}_{j}\right)=\lambda_{j} \gamma_{j}
$$

Since $u_{j}\left(\bar{x}_{j}\right)=u_{j}(\Lambda)$ does not depend on a choice of equilibrium it follows that rates of growth depend on the model only and so they coincide for all equilibria.

Using rates of growth we can represent the system (36) in the form

$$
\sum_{j \in J} \frac{1}{\gamma_{j}} \frac{\partial}{\partial \lambda_{k}} u_{j}(\Lambda)=0 \quad(k \in J) .
$$

\section{Conclusion and further research}

The results we have obtained can be applied in the study of special types of equilibrium models with fixed budgets which arise in the theory of von Neumanntype economic dynamics, in particular in the theory of models of reproduction ([7]). There are very few results concerning these models with the added complication of restrictions on exchange. Special mechanisms for the construction of trajectories for these models can be developed using models with fixed budgets where both utility functions and budgets are parameters of the models. Applying Theorems 5.1 and 5.2 it should be possible to study which parameter values guarantee the construction of efficient, or at least almost efficient, trajectories. We will, in future research, apply the results in this paper to the study of special types of models of reproduction with public goods and restrictions on exchange, in particular where the restriction is achieved by fixed price structures or by direct distribution of part of the totality of produced goods. We believe also that models with fixed budgets will be useful in the study of special equilibrium models with non-fixed budgets.

\section{Acknowledgements}

This research has been supported by a University of Ballarat Competitive Research Grant.

\section{References}

[1] K. J. Arrow and F. N. Hahn, General competitive analysis (Holden-Day, San-Francisco, 1971). 
[2] K. J. Arrow and M. D. Intrilligator (eds.), Handbook of mathematical economics 2 (North-Holland, Amsterdam, 1982).

[3] V. F. Dem'yanov and A. M. Rubinov, Quasidifferential calculus (Optimization Software, New York, 1986).

[4] J. H. Dréze and H. Müller, “Optimality properties of rationing schemes”, J. Economic Theory 23 (1980) 131-149.

[5] F. A. Gadzhiev and A. M. Rubinov, "Models of economic equilibrium in the presence of superlinear connections", Soviet Math. Dokl. 44 (1992) 757-761.

[6] J. M. Grandmont, "Temporary general equilibrium theory", Econometrica 45 (1977) 535-572.

[7] V. L. Makarov, M. I. Levin and A. M. Rubinov, Mathematical economic theory: pure and mixed types of economic mechanisms, Advanced Textbooks in Economics 33 (North-Holland, Amsterdam, 1995).

[8] H. Nikaido, Convex structures and economic theory (Academic Press, New York, 1969).

[9] V. M. Polterovich, "On stability of some resource allocation and price control processes", in Mathematical economy and functional analysis, (in Russian), (Nauka, Moscow, 1978).

[10] R. T. Rockafellar, Convex analysis (Princeton University Press, Princeton, New Jersey, 1970).

[11] A. M. Rubinov, "Equilibrium with fixed prices: coupons or budget functions?", Working paper 7/96, SITMS, University of Ballarat, 1996.

[12] A. V. Timokhov, Mathematical models of economic reproduction, (in Russian) (Moscow State University Press, Moscow, 1982). 